\title{
Light Clusters in Core-Collapse Supernovae
}

\section{G. Röpke*}

Universität Rostock, Institut für Physik, 18051 Rostock, Germany

E-mail: gerd.roepke@uni-rostock.de

Light clusters (up to $\mathrm{A}=4$ ) in nuclear matter at subsaturation densities are treated in a quantum statistical approach. Self-energy, Pauli-blocking, and effects of continuum correlations are taken into account to calculate the quasiparticle properties and abundances of light elements [1]. Results are compared with experiments from Heavy Ion Collisions [2]. Consequences for the Equation of State [3] and nuclear structure are given [4]. The appearence of light clusters in core-collapse supernovae at post-bounce stage may modify the neutrino emission and absorption processes and, thereby, influence the supernova mechanism [5].

11th Symposium on Nuclei in the Cosmos, NIC XI

July 19-23, 2010

Heidelberg, Germany

${ }^{*}$ Speaker. 


\section{Spectral Function and Nuclear Matter Equation of State}

In the low-density limit, warm nuclear matter can be described as a mixture of different components, the clusters. In equilibrium, a mass action law can be derived, and the composition is determined by the nuclear statistical equilibrium (NSE). We consider only strong interaction, not $\beta$ equilibrium due to weak interaction. Besides the temperature $T$, the total number of protons $n_{p}$ and neutrons $n_{n}$ is fixed as conserved quantities. In the grand canonical ensemble, the corresponding chemical potentials $\mu_{n}, \mu_{p}$ are introduced. The relation to the nucleon numbers is given by the equation of state (EOS)

$$
n_{\tau}\left(T, \mu_{p}, \mu_{n}\right)=\frac{2}{\Omega} \sum_{p} \int \frac{d \omega}{2 \pi} f_{\tau}(\omega) A_{\tau}(\vec{p}, \omega)
$$

where $\Omega$ denotes the system volume, the single nucleon states $\{\vec{p}, \sigma, \tau\}$ are given by the momentum $\vec{p}$, spin summation gives the factor 2 , the isospin $\tau=n, p$, the Fermi function reads $f_{\tau}(\omega)=\left[\exp \left(\left(\omega-\mu_{\tau}\right) / T\right)+1\right]^{-1}$, and $A_{\tau}(\vec{p}, \omega)$ is the spectral function.

Using many-particle theory and cluster expansion of the self-energy [1], the result for the NSE

$$
\begin{aligned}
& n_{p}\left(T, \mu_{p}, \mu_{n}\right)=\frac{1}{\Omega} \sum_{A, v, P} Z f_{A, Z}\left[E_{A, v}(P)\right], \\
& n_{n}\left(T, \mu_{p}, \mu_{n}\right)=\frac{1}{\Omega} \sum_{A, v, P}(A-Z) f_{A, Z}\left[E_{A, v}(P)\right],
\end{aligned}
$$

is obtained, where

$$
f_{A, Z}(\omega)=\left[\exp \left(\beta\left(\omega-Z \mu_{p}-(A-Z) \mu_{n}\right)\right)-(-1)^{A}\right]^{-1}
$$

is the Fermi or Bose distribution function which depends on the inverse temperature $\beta=1 /\left(k_{B} T\right)$ and the chemical potentials $\mu_{p}, \mu_{n}$ (instead of the isospin quantum number $\tau_{1}$ we use the charge number $Z$ ). The internal quantum number $v$ denotes the excited states of the cluster $A, Z$, including the continuum states. In addition to the free nucleons $A=1$, where $Z=0$ for $n$ and $Z=1$ for $p$, all higher clusters are included.

The account of continuum states is of relevance for the EOS. In particular, the second virial coefficient can be expressed in terms of the bound state energies and the scattering phase shifts corresponding to the Beth-Uhlenbeck formula for the correlated density, see [6]

$$
n_{\mathrm{corr}}\left(T, \mu_{p}, \mu_{n}\right)=\frac{1}{\Omega} \sum_{P, v} f_{2}\left(E_{2}(P)\right)+\frac{1}{\Omega} \sum_{P, v} g_{v} \int_{0}^{\infty} \frac{d E}{\pi} f_{2}\left(E+P^{2} / 4 m\right) \frac{d}{d E} \delta_{v}(E) .
$$

The internal quantum number refers to the spin and angular momentum, not relative energy. In the low-density region where cluster formation is of relevance, a cluster Beth-Uhlenbeck formula has been discussed recently [7].

The quasiparticle concept allows us to use similar relations similar for the EOS, but with dispersion relations $E_{A, v}(P)$ that depend also on temperature, and both chemical potentials. We extend the quasiparticle concept that is familiar for the single nucleon states to the light nuclei using the definition $E_{A, v}^{\mathrm{qu}}(P)=E_{A, v}^{(0)}(P)+\operatorname{Re} \Sigma_{A, v}\left(P, E_{A, v}^{\mathrm{qu}}(P)\right)$ with the $A$-particle self-energy $\Sigma_{A, v}(P, \omega)$. 
Approximations have to be performed to evaluate the self-energies. We will analyze this quantity $E_{A, v}\left(P ; T, n_{B}, Y_{p}\right)$ as a function of the baryon density $n_{B}=n_{n}+n_{p}$ and the proton fraction $Y_{p}=$ $n_{p} / n_{B}$.

For the single nucleon quasiparticle energies $E_{1}^{\mathrm{qu}}(1)$, different expressions are well established such as Skyrme, relativistic mean field or microscopic Dirac-Brueckner Hartree-Fock calculations. The few-body problem describing $A$ nucleons in hot and dense matter can be related to an inmedium wave equation (Bethe-Salpeter equation) which is derived from many-particle approaches, see Ref. [1]. The $A$-particle wave function and the corresponding eigenvalues follow from solving the in-medium Schrödinger equation

$$
\begin{aligned}
& {\left[E_{1}^{\mathrm{qu}}(1)+\ldots+E_{1}^{\mathrm{qu}}(A)-E_{A v}^{\mathrm{qu}}(P)\right] \psi_{A v P}(1 \ldots A)} \\
& +\sum_{1^{\prime} \ldots A^{\prime}} \sum_{i<j}\left[1-f_{1}(i)-f_{1}(j)\right] V\left(i j, i^{\prime} j^{\prime}\right) \prod_{k \neq i, j} \delta_{k k^{\prime}} \psi_{A v P}\left(1^{\prime} \ldots A^{\prime}\right)=0 .
\end{aligned}
$$

Here, the nucleon-nucleon interaction $V\left(i j, i^{\prime} j^{\prime}\right)$ becomes medium dependent due to the Pauli blocking prefactor $\left[1-f_{1}(i)-f_{1}(j)\right]$. The phase space occupation is described by a Fermi distribution function normalized to the total density of nucleons,

$$
f_{1}(1)=\frac{1}{\exp \left[E_{1}^{\mathrm{qu}}(1) / T-\mu_{\tau} / T\right]+1} \approx \frac{n_{\tau}}{2}\left(\frac{2 \pi \hbar^{2}}{m T}\right)^{3 / 2} e^{-E_{1}^{\mathrm{qu}}(1) / T}
$$

in the low-density, non-degenerate limit $\left(\mu_{\tau}<0\right)$. The chemical potential $\mu_{\tau}$ is determined by the normalization condition $2 \Omega^{-1} \sum_{p} f_{1}(p)=n_{\tau}$, where $\tau$ denotes isospin (neutron or proton), and has to be expressed in terms of these densities and the temperature.

The in-medium Schrödinger equation (1.5) contains the effects of the medium in the quasiparticle shift as well as in the Pauli blocking terms. Obviously the bound state wave functions and energy eigenvalues as well as the scattering phase shifts become dependent on temperature and density. We are interested in the binding energies $E_{A v}^{\text {bind }}(P)=A E_{1}^{\mathrm{qu}}(P / A)-E_{A v}^{\mathrm{qu}}(P)$ that indicates the energy difference between the bound state and the continuum of free (scattering) states at the same total momentum $P$. With increasing density, the binding energies become weaker. For given temperature, at a critical density the binding energy goes to zero. Above that, ony bound states with sufficiently large momenta can exist. An extension of the Beth-Uhlenbeck formula that includes also the medium modifications of the bound and scattering states is discussed in [6].

\section{Expressions for the Quasiparticle Shifts}

We are interested in the light clusters $v={ }^{2} \mathrm{H},{ }^{3} \mathrm{H},{ }^{3} \mathrm{He},{ }^{4} \mathrm{He}$. Using the empirical values for the binding energies and the rms point radii, a Gaussian separable interaction potential

$$
V\left(i j, i^{\prime} j^{\prime}\right)=\lambda \delta_{p_{1}+p_{2}, p_{1}^{\prime}+p_{2}^{\prime}} e^{-\frac{\left(p_{1}-p_{2}\right)^{2}}{4 \gamma^{2}}} e^{-\frac{\left(p_{1}^{\prime}-p_{2}^{\prime}\right)^{2}}{4 \gamma^{2}}}
$$

has been fitted and the in-medium Schrödinger equation has been solved, see Ref. [1].

The few-body Schrödinger equation has been solved within a variational approach. A Jastrow ansatz which reproduces the exact solution for $A=2$ for Gaussian separable interaction is given by

$$
\varphi_{v}^{\text {Jastrow }}\left(\vec{p}_{1} \ldots \vec{p}_{A}\right)=\frac{1}{N_{V}} \prod_{i<j} \frac{e^{-\frac{\left(\vec{p}_{j}-\vec{p}_{i}\right)^{2}}{4 a_{V}^{2}}}}{\frac{\left(\vec{p}_{j}-\vec{p}_{i}\right)^{2}}{4 b_{V}^{2}}+1} .
$$


Table 1: Light cluster wave function parameters at zero density from the Jastrow approach

\begin{tabular}{|l|l|l|c|l|l|l|}
\hline$v$ & $\begin{array}{l}\lambda_{v} \\
{\left[\mathrm{MeV} \mathrm{fm}^{3}\right]}\end{array}$ & $\begin{array}{l}\gamma_{v} \\
{\left[\mathrm{fm}^{-1}\right]}\end{array}$ & $\begin{array}{c}a_{v} \\
{\left[\mathrm{fm}^{-1}\right]}\end{array}$ & $\begin{array}{l}b_{v} \\
{\left[\mathrm{fm}^{-1}\right]}\end{array}$ & $\begin{array}{l}E_{b} \\
{[\mathrm{MeV}]}\end{array}$ & $\begin{array}{l}\text { rms point } \\
{[\mathrm{fm}]}\end{array}$ \\
\hline$d\left({ }^{2} \mathrm{H}\right)$ & -1287.4 & 1.474 & 1.474 & 0.2317 & -2.225 & 1.96 \\
$t\left({ }^{3} \mathrm{H}_{\text {aver }}\right.$ & -1467.03 & 1.153 & 1.5947 & 0.5670 & -8.482 & 1.683 \\
$h\left({ }^{3} \mathrm{He}\right)_{\text {aver }}$ & -1431.85 & 1.153 & 1.602 & 0.5514 & -7.718 & 1.713 \\
$\alpha\left({ }^{4} \mathrm{He}\right)$ & -1272.9 & 1.231 & 2.151 & 0.912 & -28.30 & 1.45 \\
\hline
\end{tabular}

The prefactor $N_{v}$ is determined by the normalization condition. The parameter values for the interaction potential and the Jastrow wave functions at zero density are shown in Tab. 1. Within a more detailed approach, different orbitals have to be considered for the protons and neutrons in the case $A=3$.

\section{Binding energies at finite densities}

The shift of energy eigenvalues in a dense medium is determined by different effects, the Coulomb interaction, the self-energy shift due to the single-nucleon quasiparticle shifts, and the Pauli blocking shift that determines the shift of the binding energies of nuclei relative to the continuum. The Pauli blocking shift results from the energy eigenvalue equation where only the Pauli blocking terms in the potential are taken into account, $\delta E_{v}^{\text {Pauli }}(P)=E_{v}^{\text {Pauli }}(P)-E_{v}^{0}(P)$, being a function of the center-of-mass momentum $P$ of the cluster, the temperature $T$, the nucleon density $n_{B}$ and the proton fraction $Y_{p}$. The Pauli blocking shift was calculated by variational approaches, but in the low-density limit by perturbation theory.

The $P$ dependence of the Pauli blocking shift is approximated by

$$
\Delta E_{v}^{\text {Pauli }}(P) \approx \Delta E_{v}^{\text {Pauli }}(0) \exp \left(-\frac{P^{2}}{\hbar^{2} g_{v}}\right)
$$

Here,

$$
\Delta E^{\text {Pauli }}(0)=\frac{n}{T^{3 / 2}} \frac{a_{v, 1}}{\left(1+a_{v, 2} / T\right)^{3 / 2}} f^{(0)}\left(y_{v}\right) \frac{1}{1+\left(b_{v, 1}+b_{v, 2} / T\right) n},
$$

where $f^{(0)}(y)=2 y^{2}\left(1-\pi^{1 / 2} y e^{y^{2}} \operatorname{Erfc}[y]\right)$ with $\lim _{y \rightarrow \infty} f^{(0)}(y)=1-3 /\left(2 y^{2}\right) \pm \ldots, \lim _{y \rightarrow 0} f^{(0)}(y)=$ $2 y^{2}\left(1-\pi^{1 / 2} y+2 y^{2}-\pi^{1 / 2} y^{3} \pm \ldots\right)$, and $y_{v}=a_{v, 3}\left(1+a_{v, 2} / T\right)^{1 / 2}$. This is exact for the two-nucleon case. The dispersion can be calculated from

$$
g_{v}\left(T, n, Y_{p}\right)=\frac{g_{v, 1}+g_{v, 2} T+h_{v, 1} n}{1+h_{v, 2} n}
$$

Values for $a_{v, i}$ are given below in Tab. 2, for the remaining parameters see Ref. [1].

Now, the nucleon number densities can be evaluated as in the non-interacting case, with the only difference that the number densities of the particles are calculated with the quasiparticle energies. In the light cluster-quasiparticle approximation, the total densities of neutrons 
Table 2: Pauli blocking shift parameter, low-density limit

\begin{tabular}{|l|l|l|c|}
\hline$v$ & $\begin{array}{l}a_{v, 1} \\
{\left[\mathrm{MeV} \mathrm{fm}^{3}\right]}\end{array}$ & $\begin{array}{l}a_{v, 2} \\
{[\mathrm{MeV}]}\end{array}$ & $a_{v, 3}$ \\
\hline$d\left({ }^{2} \mathrm{H}\right)$ & 388338 & 22.52 & 0.2223 \\
$t\left({ }^{3} \mathrm{H}\right)_{\text {aver }}$ & 159227 & 11.99 & 0.8539 \\
$h\left({ }^{3} \mathrm{He}\right)_{\text {aver }}$ & 155014 & 11.84 & 0.8351 \\
$\alpha\left({ }^{4} \mathrm{He}\right)$ & 553217 & 22.10 & 0.6689 \\
\hline
\end{tabular}

$n_{n}=n_{n}^{\text {free }}+\sum_{v=d, t, h, \alpha} N_{v} n_{v}$ and of protons $n_{p}=n_{p}^{\text {free }}+\sum_{v=d, t, h, \alpha} Z_{v} n_{v}$ contain the densities of the free neutrons and protons $n_{n}^{\text {free }}$ and $n_{p}^{\text {free }}$, respectively, and the contributions from the nucleons bound in the clusters with densities $n_{v}$.

\section{Results}

From the equation of state $n\left(T, \mu_{p}, \mu_{n}\right)$, thermodynamic potentials are obtained by integration, and other thermodynamic properties can be derived in a consistent way. Results are given in [3]. The composition has been calculated for subsaturation densities, and it has been shown that bound states disappear with increasing density due to the Pauli blocking effect. As example, the symmetry energy can be derived from the measured cluster yields in Heavy Ion Collisions [2] using the quantum statistical approach to calculate light clusters in nuclear matter given here. Another example for clustering in low-density matter are excited nuclei such as the Hoyle state [4]. Both examples can be considered as experimental checks for the in-medium behavior of light elements.

Of particular interest is the calculation of clusters in core-collapse supernovae [5] that demands the in-medium treatment of light clusters. The formation of correlations, in particular bound states, is of crucial importance for different transport processes such as neutrino emission and absorption. The quasiparticle approach and the evaluation of the in-medium shifts, see Tab. 2, are indispensable prerequisites to investigate the abundances of light clusters in nuclear matter.

\section{Acknowledgments:}

This work was supported by the Deutsche Forschungsgemeinschaft, project RO905/30-1.

\section{References}

[1] G. Röpke, Phys. Rev. C79, 014002 (2009).

[2] J. Natowitz et al., Phys. Rev. Lett. 104, 202501 (2010).

[3] S. Typel et al., Phys. Rev. C81, 015803 (2010).

[4] Y. Funaki et al., Phys. Rev. C80, 064326 (2009).

[5] K. Sumiyoshi and G. Röpke, Phys. Rev. C77, 055804 (2008).

[6] M. Schmidt et al., Ann. Phys. 202, 57 (1990).

[7] C. J. Horowitz, A. Schwenk, Nucl. Phys. A776 55 (2006). 